\title{
Geometric Error Modeling and Sensitivity Analysis of CNC Internal Circular Compound Grinding Machine
}

\author{
Jinwei Fan', Qiang Liu", *, Weihua Li², Liangliang Xue', Chenbao Li ${ }^{1}$ \\ ${ }^{1}$ School of Mechanical Engineering and Applied Electronics Technology, Beijing University of Technology, Beijing, China \\ ${ }^{2}$ Machinery Department, Beijing Second Machine Tool Factory Co. Ltd, Beijing, China \\ Email address: \\ jwfan@bjut.edu.cn (Jinwei Fan), 1396797897@qq.com (Qiang Liu), liweihua1974@163.com (Weihua Li), \\ xueliangliang1996@163.com (Liangliang Xue),718052067@qq.com (Chenbao Li) \\ ${ }^{*}$ Corresponding author
}

\section{To cite this article:}

Jinwei Fan, Qiang Liu, Weihua Li, Liangliang Xue, Chenbao Li. Geometric Error Modeling and Sensitivity Analysis of CNC Internal Circular Compound Grinding Machine. International Journal of Mechanical Engineering and Applications. Vol. 8, No. 5, 2020, pp. 118-124. doi: 10.11648/j.ijmea.20200805.12

Received: September 21, 2020; Accepted: October 5, 2020; Published: October 27, 2020

\begin{abstract}
In order to improve the machining accuracy and efficiency of the hole and sleeve parts, it is necessary to improve the overall grinding accuracy of the CNC (Computer Numerical Control) internal cylindrical compound grinding machine more accurately and efficiently. First of all, it is necessary to clarify the degree of influence of each error parameter on the grinding accuracy, and compensate each error according to the different degree of influence. In this paper, modeling calculation analysis is carried out for a certain type of CNC internal cylindrical compound grinding machine. Firstly, based on the theory of multi-body system dynamics, the topological structure of the $\mathrm{CNC}$ internal cylindrical compound grinder is established. According to the topological structure, the position, motion matrix and error matrix of the moving parts of the grinder are written. After data processing, the numerical control internal cylindrical compound grinder is calculated. Use this model to derivate each error parameter to obtain the sensitivity expression of each error parameter. After the actual structure parameters of the grinder are brought into the expression, the sensitivity coefficient of each error parameter can be determined by normalization treatment. The key error parameter with larger sensitivity coefficient is the key error parameter. Finally, several error parameters which have the greatest impact on the overall grinding accuracy of the grinder are obtained. This method provides the basis for the improvement of the grinding accuracy of the subsequent grinder, and creates conditions for the improvement of the machining accuracy of sleeve parts.
\end{abstract}

Keywords: Multi-body Theory, Geometric Error, Sensitivity Coefficient, Accuracy Improvement

\section{Introduction}

The hole is one of the important surfaces of the disc and sleeve parts. The hole at the axis position of the shaft, disc and sleeve is generally processed by lathe and grinder. The high-precision hole on the sleeve metal parts is mostly processed by the internal grinder [1]. The sleeve parts are grinded by $\mathrm{CNC}$ Internal compound grinder. After grinding the inner hole, the outer circle can be ground, which greatly improves the machining efficiency. In the mechanical processing industry, the internal grinder is often used for high-precision grinding, so there is a high demand for the grinding accuracy of the grinder. The geometric error analysis model is established for the internal compound grinder, and the sensitivity analysis of each error parameter contained in the model is carried out. According to the analysis results, the sensitive error parameters are compensated to improve the grinding accuracy of the grinder.

In terms of mathematical modeling and model analysis, sensitivity analysis can provide guidance for follow-up work, so researchers all regard sensitivity analysis as a prerequisite for modeling and model analysis [2]. At present, the commonly used sensitivity analysis methods include local sensitivity analysis and global sensitivity analysis [3, 4]. Wu Jian [5] established the error model of the VMC850B machine tool using the homogeneous coordinate transformation method, and quantified the influence of each error parameter on the overall machining error through global 
sensitivity analysis. K. Fan [6] proposed a sensitivity analysis method to identify the most sensitive parameters that affect the position and direction of the parallel mechanism, and effectively identified the most sensitive parameters. Zhao $\mathrm{Yu}$ [7] proposed a set of modeling process for machine tool spatial error, and based on the theory of multi-body system, established the spatial error model of milling and boring five-axis machining center. Yang Chengxu et al. [8] established the spatial error model of the four-axis motion platform based on the theory of multi-body systems. Andreas Pott [9] analyzed the sensitivity of the parallel mechanism with the simplified vector force method and verified it by experiments. Xiao Longfan [10] proposed a sensitivity identification method based on the multi-body system and the linear response surface method to analyze the accuracy of the machine tool. And applied the 5-axis horizontal CNC milling machine. Kang Yueran [11] proposed a method for identifying key error sources of three-axis turntable based on sensitivity analysis. Taking a three-axis simulation turntable as the research object, the key error sources that have a greater impact on the pointing error of the three-axis turntable are identified. Li et al. [12] analyzed the degree of influence of each geometric error source on the spatial error model of the five-axis machining center, and obtained the key components that affect the machining error of the machine tool, and verified the effectiveness of the sensitivity analysis method through digital simulation. Fan [13] used a five-axis machine tool machining error global sensitivity analysis method based on quasi-Monte Carlo algorithm to evaluate the degree of influence of various geometric error parameters on the machine tool machining error model.

Comprehensive analysis, this paper uses the multi-body system theory to establish the geometric error model of the internal grinder, and through the error sensitivity analysis, obtains the influence of the error parameters on the machining error, and quantifies the results, which provides the basis for the subsequent targeted reduction of the machining error of the internal grinder.

\section{Structure Analysis of CNC Internal Grinder}

The internal cylindrical compound grinder can improve the processing efficiency of sleeve parts, which is the main direction of development [14]. The internal composite grinder is mainly used for the internal machining of parts, and can also be used for grinding the external cylindrical surface. The cylindrical parts with small size can also be grinded by the internal composite grinder. In this paper, the CNC Internal compound grinder produced by a factory is taken as the research object. The structure of the grinder is shown in Figure 1. The brick tower of the grinding spindle of the compound grinder can be equipped with four spindles, including internal grinding spindle, external grinding spindle and measuring probe, and the built-in rotary shaft drives the turret to rotate. The workpiece can be processed completely after one clamping, which can not only improve the accuracy but also improve the processing efficiency.

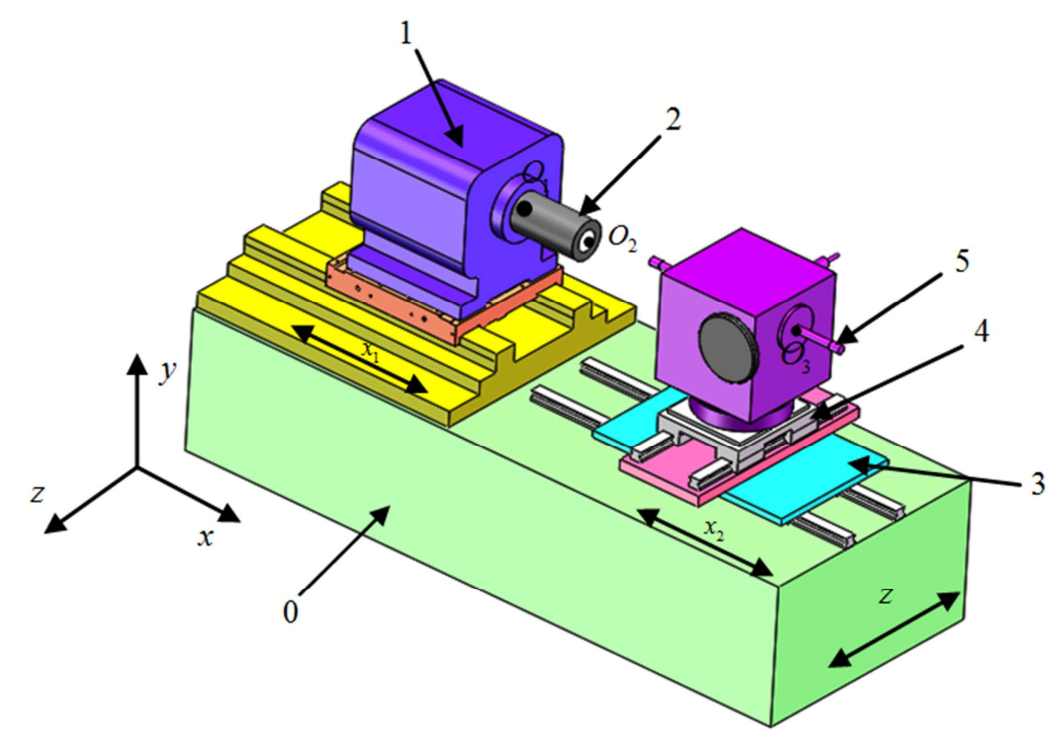

0-bed; 1-headstock; 2-workpiece; 3-x2 slide seat; 4-z slide seat; 5-spindle brick tower grinding wheel spindle;

Figure 1. Structure diagram of CNC Internal Grinder.

\section{Error Parameter Analysis of CNC Internal Grinder}

The larger grinding wheel on the main shaft brick tower is cylindrical wheel, which is used to grind shaft parts. After rotating, the smaller grinding wheel is the inner round wheel to grind the inner circle parts. In the grinding process, the $\mathrm{x} 1$ sliding seat drives the head frame along $\mathrm{x}$ There are six spatial errors in each motion axis, including three linear displacement errors and three angular displacement errors. There are also non perpendicularity errors between the vertical $y$-axis and 
$\mathrm{X}$-Z-axis The error has a certain influence on the grinding accuracy, which needs to be considered when analyzing the error parameters.

The error parameters of the compound grinder are shown in Table 1 . The axis of the head frame is $\mathrm{x} 1$ axis, and the axis of the main shaft brick tower is $\mathrm{x} 2$ axis.

Table 1. Geometric error parameters of CNC Internal Grinder.

\begin{tabular}{lll}
\hline Error properties & $\begin{array}{l}\text { Linear } \\
\text { displacement error }\end{array}$ & $\begin{array}{l}\text { Angular } \\
\text { displacement error }\end{array}$ \\
\hline $\begin{array}{l}\text { Translation along X1 axis } \\
\text { Translation along the } \mathrm{X} 2\end{array}$ & $\delta_{\mathrm{x}}(\mathrm{x} 1) \delta_{\mathrm{y}}(\mathrm{x} 1) \delta_{\mathrm{z}}(\mathrm{x} 1)$ & $\varepsilon_{\mathrm{x}}(\mathrm{x} 1) \varepsilon_{\mathrm{y}}(\mathrm{x} 1) \varepsilon_{\mathrm{z}}(\mathrm{x} 1)$ \\
$\begin{array}{l}\text { axis } \\
\begin{array}{l}\text { Translation along the } \\
\text { Z-axis } \\
\text { verticality }\end{array}\end{array}$ & $\delta_{\mathrm{x}}(\mathrm{x} 2) \delta_{\mathrm{y}}(\mathrm{x} 2) \delta_{\mathrm{z}}(\mathrm{x} 2)$ & $\varepsilon_{\mathrm{x}}(\mathrm{x} 2) \varepsilon_{\mathrm{y}}(\mathrm{x} 2) \varepsilon_{\mathrm{z}}(\mathrm{x} 2)$ \\
\hline
\end{tabular}

Table 1 geometric error parameters of CNC Internal compound grinding machine this paper takes the internal grinding of the compound grinder as an example to carry out geometric error modeling, and carry out sensitivity analysis to obtain the sensitivity coefficient of each error parameter, and find the key error items.

\section{Establishment of Geometric Error Model for Grinder}

\subsection{Establishment of Grinding Machine Topology}

Multi-body system refers to a complex mechanical system composed of multiple rigid or flexible bodies connected in some form [15]. Establishing the topological structure of the grinder based on the theory of the multi-body system makes it easier to describe the multi-body system with a computer, and the calculation is simple. Based on the theory of multi-body system, the topological structure of this compound grinder is analyzed. The grinder is a multi-body system with two branches, namely the "bed-workpiece" branch and the "bed-tool" branch. The topology is shown in Figure 2 below.

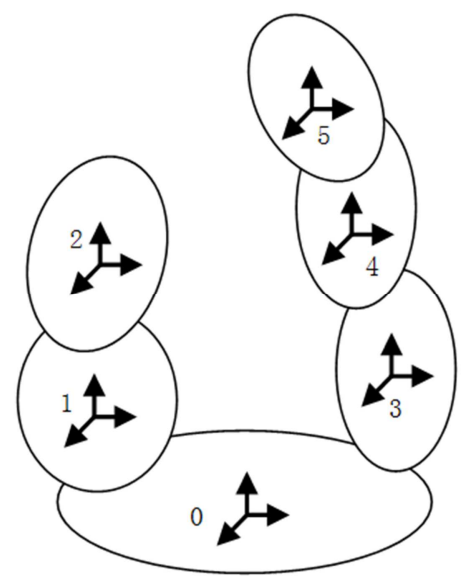

Figure 2. Topological structure diagram of internal composite grinder.

Suppose a grinding point on the workpiece is point $\mathrm{P}$, and the position matrix and displacement matrix are used to describe the point $\mathrm{P}$ to the inertial coordinate system along two branches respectively. The matrix expression along the "bed-workpiece" branch is:

$$
\begin{aligned}
\left\{P_{w}\right\}_{0}= & {[S 01]_{p}[S 01]_{p e}[S 01]_{S}[S 01]_{s e}[S 12]_{p}[S 12]_{p e}[S 12]_{s} } \\
& {[S 12]_{s e} *\left\{r_{w}\right\}_{w} }
\end{aligned}
$$

The matrix expression along the "bed-tool" branch is :

$$
\begin{gathered}
\left\{P_{t}\right\}_{0}=[S 03]_{p}[S 03]_{p e}[S 03]_{s}[S 03]_{s e}[S 34]_{p}[S 34]_{p e}[S 34]_{s} \\
{[S 34]_{s e}[S 45]_{p}[S 45]_{p e}[S 45]_{S}[S 45]_{s e} *\left\{r_{t}\right\}_{t}}
\end{gathered}
$$

Where: $\left\{r_{w}\right\}_{w}$ is the position matrix expression of point $\mathrm{P}$ in the workpiece coordinate system;

$\left\{r_{t}\right\}_{t}$ is the position matrix expression of point $\mathrm{P}$ in the tool coordinate system;

In the grinding process of the grinder, the grinding point on the grinding wheel needs to coincide with the position of the ground point on the workpiece in the inertial coordinate system, then the constraint equation is $\left\{P_{w}\right\}_{0}=\left\{P_{t}\right\}_{0}$, a coordinate system is established for each part of the machine tool, and the corresponding transformation matrix can be obtained for solving.

\subsection{The Establishment of Moving Body Coordinate System and Error Matrix}

To establish a geometric error model, it is necessary to determine the position matrix and motion matrix of each part of the grinder, so the corresponding coordinate system of each part needs to be established first. Move each part of the machine tool to the initial position so that the body reference coordinate system of each part of the machine tool coincides with the respective motion reference coordinate system, that is, under the initial conditions, the body reference coordinate system can be determined according to the motion reference coordinate system of a certain part position. The orientation of the spatial coordinate system involves the direction and position, and the reference direction of the coordinate system of each component is selected as the direction of the basic coordinate system of the machine tool. The position of the coordinate system of each part is set separately. First, the parts of the machine tool are moved to the absolute zero point of the machine tool, and the volume reference coordinate system of the workpiece and the grinding wheel are set at the center of the workpiece end face $\mathrm{O}_{2}$ and the end face center $\mathrm{O}_{3}$ of the grinding wheel respectively. The positions of the body reference coordinate system are all at the center $\mathrm{O}_{1}$ of the end face of the headstock spindle. Taking into account the non-perpendicularity error of the coordinate axis of each component, the basic coordinate system of the machine tool is rotated around its $y$ and $z$ axes through the perpendicularity $\varepsilon_{\mathrm{x} 1 \mathrm{y}}$ and $\varepsilon_{\mathrm{x} 1 \mathrm{z}}$. The direction of the coordinate system is the direction of the body reference coordinate system of the headstock spindle. The direction of the coordinate system after the basic coordinate system is rotated 
around its $\mathrm{x}$ and $\mathrm{y}$ axes through the perpendicularity $\varepsilon_{\mathrm{x} 2 \mathrm{z}}$ and $\varepsilon_{\mathrm{yz}}$ is the direction of the body reference coordinate system of the grinding wheel spindle. Considering the structure of the grinder, the headstock and the x1-direction worktable are regarded as one body, and $\mathrm{x} 2$ The sliding plate and the spindle turret are regarded as one body. Suppose the position matrix of the workpiece coordinate system relative to the center point $\mathrm{O}_{1}$ of the headstock spindle is (q2x q2y q2z 1) T, and the position matrix of the grinding wheel coordinate system relative to the center point $\mathrm{O}_{1}$ of the headstock spindle is ( $95 \mathrm{x}$ q5y q5z 1) T. According to the above analysis, the position and motion transformation matrix between the parts of the grinder is

$$
\begin{aligned}
& {[\mathrm{S} 01]_{p}=E \quad[\mathrm{~S} 01]_{p e}=E} \\
& {[\mathrm{~S} 01]_{S}=\left[\begin{array}{cccc}
1 & 0 & 0 & x 1 \\
0 & 1 & 0 & 0 \\
0 & 0 & 1 & 0 \\
0 & 0 & 0 & 1
\end{array}\right]} \\
& {[S 01]_{s e}=\left[\begin{array}{cccc}
1 & -\varepsilon_{z}(x 1) & \varepsilon_{y}(x 1) & \delta_{x}(x 1) \\
\varepsilon_{z}(x 1) & 1 & -\varepsilon_{x}(x 1) & \delta_{y}(x 1) \\
-\varepsilon_{y}(x 1) & \varepsilon_{x}(x 1) & 1 & \delta_{z}(x 1) \\
0 & 0 & 0 & 1
\end{array}\right]} \\
& {[\mathrm{S} 12]_{p}=\left[\begin{array}{cccc}
1 & 0 & 0 & q_{2 x} \\
0 & 1 & 0 & q_{2 y} \\
0 & 0 & 1 & q_{2 z} \\
0 & 0 & 0 & 1
\end{array}\right]} \\
& {[\mathrm{S} 12]_{p e}=\left[\begin{array}{cccc}
1 & -\varepsilon_{x 1 y} & \varepsilon_{x 1 z} & 0 \\
\varepsilon_{x 1 y} & 1 & 0 & 0 \\
-\varepsilon_{x 1 z} & 0 & 1 & 0 \\
0 & 0 & 0 & 1
\end{array}\right]} \\
& {[\mathrm{S} 12]_{S}=E \quad[\mathrm{~S} 12]_{s e}=E} \\
& {[\mathrm{~S} 03]_{p}=E \quad[\mathrm{~S} 03]_{p e}=E} \\
& {[\mathrm{~S} 03]_{S}=\left[\begin{array}{cccc}
1 & 0 & 0 & x 2 \\
0 & 1 & 0 & 0 \\
0 & 0 & 1 & 0 \\
0 & 0 & 0 & 1
\end{array}\right]} \\
& {[S 03]_{s e}=\left[\begin{array}{cccc}
1 & -\varepsilon_{z}(x 2) & \varepsilon_{y}(x 2) & \delta_{x}(x 2) \\
\varepsilon_{z}(x 2) & 1 & -\varepsilon_{x}(x 2) & \delta_{y}(x 2) \\
-\varepsilon_{y}(x 2) & \varepsilon_{x}(x 2) & 1 & \delta_{z}(x 2) \\
0 & 0 & 0 & 1
\end{array}\right]}
\end{aligned}
$$

$$
\begin{aligned}
& {[\mathrm{S} 34]_{p}=E[\mathrm{~S} 34]_{p e}=\left[\begin{array}{cccc}
1 & 0 & \varepsilon_{x 2 z} & 0 \\
0 & 1 & -\varepsilon_{y z} & 0 \\
-\varepsilon_{x 2 z} & \varepsilon_{y z} & 1 & 0 \\
0 & 0 & 0 & 1
\end{array}\right]} \\
& {[\mathrm{S} 34]_{S}=\left[\begin{array}{cccc}
1 & 0 & 0 & 0 \\
0 & 1 & 0 & 0 \\
0 & 0 & 1 & z \\
0 & 0 & 0 & 1
\end{array}\right]} \\
& {[S 34]_{s e}=\left[\begin{array}{cccc}
1 & -\varepsilon_{z}(z) & \varepsilon_{y}(z) & \delta_{x}(z) \\
\varepsilon_{z}(z) & 1 & -\varepsilon_{x}(z) & \delta_{y}(z) \\
-\varepsilon_{y}(z) & \varepsilon_{x}(z) & 1 & \delta_{z}(z) \\
0 & 0 & 0 & 1
\end{array}\right]} \\
& {[\mathrm{S} 45]_{p}=\left[\begin{array}{cccc}
1 & 0 & 0 & q_{5 x} \\
0 & 1 & 0 & q_{5 y} \\
0 & 0 & 1 & q_{5 z} \\
0 & 0 & 0 & 1
\end{array}\right][\mathrm{S} 45]_{p e}=E} \\
& {[\mathrm{~S} 45]_{s}=E \quad[\mathrm{~S} 45]_{s e}=E}
\end{aligned}
$$

\subsection{Geometric Error Model Solution}

According to the grinding conditions, the grinding wheel and the workpiece are tangent at the grinding point, which can be obtained

$$
\begin{gathered}
\left\{r_{w}\right\}_{w}=\left\{\begin{array}{llll}
x_{w} & 0 & R & 1
\end{array}\right\}^{T} \\
\left\{r_{t}\right\}_{t}=\left\{\begin{array}{lllll}
-L & 0 & r & 1
\end{array}\right\}^{T}
\end{gathered}
$$

Where: $\mathrm{R}$ is the radius of the workpiece, $\mathrm{r}$ is the radius of the grinding wheel, and $\mathrm{xw}$ is the distance between the grinding point and the center of the spindle end face of the headstock. $\mathrm{L}$ is the distance from the outer end surface of the grinding wheel to the $\mathrm{O}_{3}$ point.

Bring the matrix obtained above into equations (1) and (2), use MATLAB software to solve the results of the two expressions, sort the results, ignoring the high-order infinitesimals, and obtain the grinding point $\mathrm{P}$ along the workpiece branch and tool The branch is described to the expression in the inertial coordinate system, and the components in the two expressions are respectively corresponding and equal. Simplification can obtain the position $\left\{P_{\text {actual }}\right\}$ of the grinding point $\mathrm{P}$ of the grinding wheel in the workpiece coordinate system under actual conditions.

The motion error parameter and position error parameter in the expression are omitted, and after simplification, the position of the grinding wheel grinding point $\mathrm{P}$ in the workpiece coordinate system $\left\{P_{\text {ideal }}\right\}$ can be obtained under ideal conditions. The geometric error model expression can 
be obtained by making the difference between the actual position matrix and the ideal position matrix.

$$
\begin{aligned}
& E=\left\{P_{\text {actual }}\right\}-\left\{P_{\text {ideal }}\right\}=\left(E_{x} E_{y} E_{z}\right) \\
& *\left(\delta_{y}(z)+q_{5 y}\right)+L *\left(\varepsilon_{x 2 z}+\varepsilon_{y}(x 2)+\varepsilon_{y}(z)\right)
\end{aligned}
$$$$
E_{x}=\delta_{x}(x 2)-\delta_{x}(x 1)+\delta_{x}(z)+\delta_{y}(z)+q_{5 y}+q_{5 z} *\left(\varepsilon_{x 2 z}+\varepsilon_{y}(x 2)+\varepsilon_{y}(z)\right)-r *\left(\varepsilon_{x}(x 2)+\varepsilon_{x}(z)+\varepsilon_{y z}\right)
$$$$
+r *\left(\varepsilon_{x 2 z}+\varepsilon_{y}(x 2)+\varepsilon_{y}(z)\right)-\left(\varepsilon_{x 1 z}+\varepsilon_{y}(x 1)\right) *\left(q_{5 z}+r+z\right)-\varepsilon_{x 1 y} * q_{2 y}+\varepsilon_{x 1 z} * q_{2 z}-L *\left(\varepsilon_{z}(x 2)+\varepsilon_{z}(z)\right)
$$$$
+q_{5 x} *\left(\varepsilon_{z}(x 2)+\varepsilon_{z}(z)\right)-q_{5 y} *\left(\varepsilon_{z}(x 2)+\varepsilon_{z}(z)\right)+z *\left(\varepsilon_{x 2 z}+\varepsilon_{y}(x 2)\right)-z *\left(\varepsilon_{x}(x 2)+\varepsilon_{y z}\right)
$$$$
E_{y}=\delta_{y}(x 2)-\delta_{y}(x 1)+\delta_{y}(z)-q_{5 z} *\left(\varepsilon_{x}(x 2)+\varepsilon_{x}(z)+\varepsilon_{y z}\right)-r *\left(\varepsilon_{x}(x 2)+\varepsilon_{x}(z)+\varepsilon_{y z}\right)
$$$$
+\varepsilon_{x 1 y} * q_{2 x}+\varepsilon_{x 1 y} * x 1+\varepsilon_{z}(x 1) * x 1-\left(\varepsilon_{x 1 y}+\varepsilon_{z}(x 1)\right) *\left(q_{5 x}-L+x 2\right)+\varepsilon_{x}(x 1) *\left(q_{5 z}+r+z\right)
$$$$
-L *\left(\varepsilon_{z}(x 2)+\varepsilon_{z}(z)\right)+q_{5 x} *\left(\varepsilon_{z}(x 2)+\varepsilon_{z}(z)\right)-z *\left(\varepsilon_{x}(x 2)+\varepsilon_{y z}\right)
$$$$
E_{z}=\delta_{z}(x 2)-\delta_{z}(x 1)+\delta_{z}(z)+q_{5 y} *\left(\varepsilon_{x}(x 2)+\varepsilon_{x}(z)+\varepsilon_{y z}\right)-q_{5 x} *\left(\varepsilon_{x 2 z}+\varepsilon_{y}(x 2)+\varepsilon_{y}(z)\right)
$$$$
-\varepsilon_{x 1 z} * q_{2 x}-\varepsilon_{x 1 z} * x 1-\varepsilon_{y}(x 1) * x 1+\left(\varepsilon_{x 1 z}+\varepsilon_{y}(x 1)\right) *\left(\delta_{x}(z)-L+q_{5 x}+x 2\right)-\varepsilon_{x}(x 1)
$$
processing.

\section{Geometric Error Sensitivity Analysis}

\subsection{Error Sensitivity Model Establishment}

The geometric error model of the internal composite grinding machine contains 22 error parameters. In order to analyze the influence of each error parameter on the final error, sensitivity analysis of each parameter is required. According to the geometric error model obtained above, it can be expressed as a function of 22 error parameters in the following form:

$$
E=f\left(\delta_{i}(j), \quad \varepsilon_{i}(j), \quad \varepsilon_{x 1 z}, \quad \varepsilon_{x 1 y}, \quad \varepsilon_{y z}, \quad \varepsilon_{x 2 z}\right)
$$

In the formula, $i=x, y, z ; j=x 1, x 2, z$;

This article uses the method of obtaining the partial derivative of each error parameter to obtain the sensitivity expression of the error parameter, and defines the sensitivity of each error parameter as $S_{u v}$.

$$
S_{u v}=\left|\partial E_{u} / \partial e_{v}\right|
$$

In the formula, $u=x, y, z ; v=1,2,3 \ldots . .22 ; e v$ is the above error items;

\subsection{Error Sensitivity Results}

In order to more intuitively express the size of each error sensitivity and the degree of influence on the overall error, the sensitivity of each error item is normalized, and the error sensitivity coefficient $\mathrm{C}$ is introduced. The value of $\mathrm{C}$ represents the degree of influence on the overall error.

$$
C=S_{u v} / \sum S_{u v}
$$

When using an example to analyze, select the grinder structure data used when processing a certain size of parts, the inner circle size of the part to be processed is $\varphi 80 \mathrm{~mm}$, and the inner circle grinding wheel size is $\varphi 40 \mathrm{~mm}$, Combined with the setting of the multi-body system coordinate system, the position of the workpiece coordinate system relative to the center point $\mathrm{O} 1$ of the headstock spindle $(\mathrm{q} 2 \mathrm{x}, \mathrm{q} 2 \mathrm{y}, \mathrm{q} 2 \mathrm{z}$, $1)=(200,0,0,1)$, the grinding wheel coordinate system is relative to the headstock spindle The position of the center point $\mathrm{O} 1$ (q6x, q6y, q6z, 1) $=(300,0,0,1)$, Randomly select a working position $(-100,0,40)$ in the processing movement area of the composite internal grinder, the error parameter value is uniformly taken as the linear error is $0.001 \mathrm{~mm}$, and the angle error is $0.001^{\circ}$. Bring the relevant values into the sensitivity expressions obtained by formula (5), the sensitivity value of each error parameter is obtained by calculation, and all the results are brought into formula (6) for normalization processing to obtain the sensitivity coefficient value, among which the key error The sensitivity values of the parameters are shown in Figures 3, 4, and 5.

It can be seen from the above results that the x-direction influences the larger error parameters $\varepsilon_{z}(x 2)$ and $\varepsilon_{z}(z)$, 
which account for nearly $25 \%$ of the total $x$-direction error, and the y-direction influences the larger error parameters $\varepsilon_{z}(x 1), \varepsilon_{z}(x 2), \varepsilon_{z}(z)$, which respectively account for the y-direction total error $29 \%, 20 \%$, and $20 \%$ of the error parameters in the $\mathrm{z}$ direction are $\varepsilon_{y}(x 1), \varepsilon_{y}(x 2), \varepsilon_{y}(z)$ and $\varepsilon_{x 2 z}$ which account for $30 \%, 20 \%, 20 \%$, and $20 \%$ of the total error in the $\mathrm{z}$ direction, respectively.

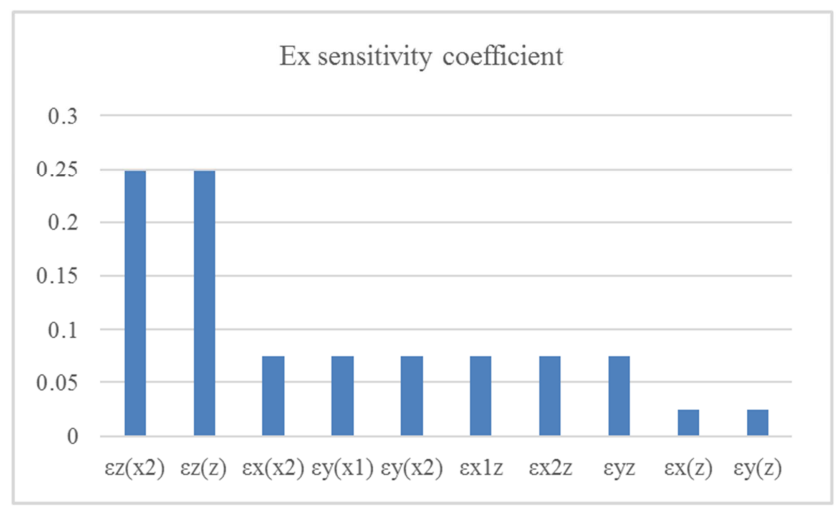

Figure 3. Sensitivity coefficient of key error parameters in the $x$ direction.

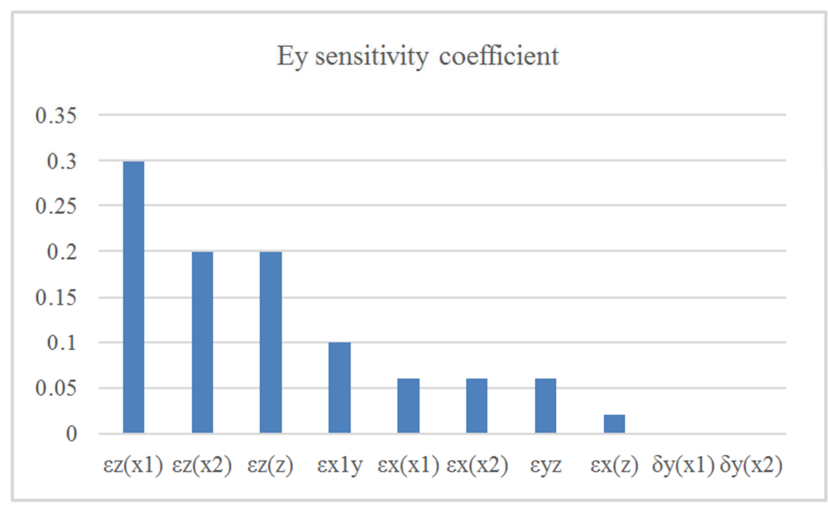

Figure 4. Sensitivity coefficients of key error parameters in $Y$ direction.

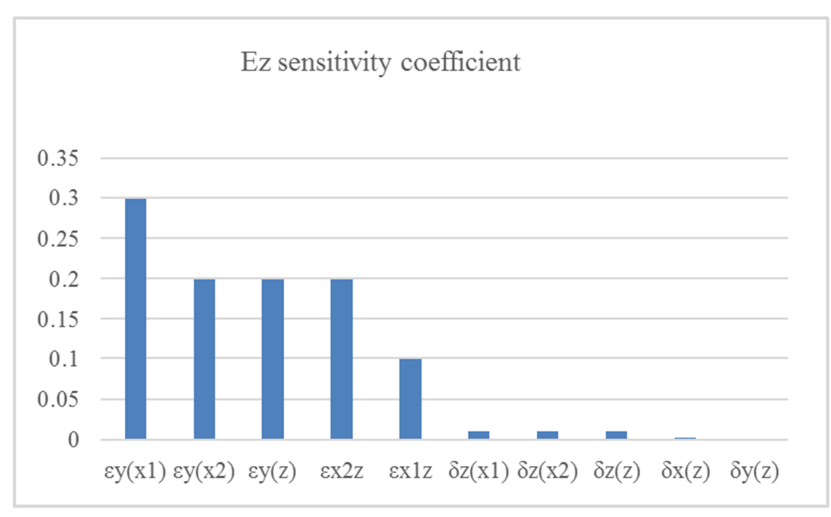

Figure 5. Sensitivity coefficients of key error parameters in z direction.

The larger sensitivity coefficients are the angle error and the verticality error of each axis. Therefore, priority should be given to the subsequent error compensation process. When adjusting the geometric accuracy of the internal grinding machine, the verticality between each axis should be adjusted. Degree relationship and reduce the yaw angle of the respective axis.

\section{Conclusion}

(1) According to the structural characteristics of the CNC internal grinding machine and the multi-body system theory, the topological structure of the internal grinding machine is established, and its geometric error model is calculated.

(2) According to the geometric error model, the sensitivity expression of each error parameter is calculated, and after the structure parameter of the grinder is brought into, the sensitivity coefficient value of each error parameter is obtained after normalization.

(3) The key error parameters that affect the machining accuracy of the grinding machine are analyzed and obtained, which provides a direction for the error compensation and geometric accuracy of the internal grinding machine, and creates conditions for the realization of the internal composite grinding machine for the finishing of sleeve parts.

\section{Acknowledgements}

This article is supported by major national science and technology projects of China (2019ZX04006-001).

\section{References}

[1] Luo Liping, Jing Yuhai. Fundamentals of mechanical manufacturing, Vol. 2 [M]. Tsinghua University Press, 2004.

[2] Saltelli A, Chan K, MarianSeott E. SensitivityAnalysis [M]. NewYork: John WileY \& Sons, Ltd, 2000. I. S. Jacobs and C. P. Bean, "Fine particles, thin films and exchange anisotropy," in Magnetism, vol. III, G. T. Rado and H. Suhl, Eds. New York: Academic, 1963, pp. 271-350.

[3] Hamby D M. A review of sensitivity analysis techniques [R]. Westinghouse Savannah River Co., Aiken, SC (United States), 1993.

[4] Borgonovo E. Sensitivity Analysis [M]. Springer, Cham, Switzerland, 2017.

[5] Wu Jian, Gu Chengjie, Du Zhengchun, et al. Error identification analysis of key components of vertical machining center [J]. Mechanical design and research, 2018, 34 (4): 81-84.

[6] K. Fan, H. Wang, J. Zhao, T. Chang, Sensitivity analysis of the 3-PRS parallel kinematic spindle platform of a serial-parallel machine tool, Int. J. Mach. Tools Manuf 43 (2003) 1561-1569.

[7] Zhao Yu, Li Tiemin, Tang Xiaoqiang, Geometric Error modeling of machine tools based on screw theory [J]. Procedia Engineering. 2011 (24): 845-849. Kass, R. E. and A. E. Raftery (1995). Bayes Factors. Journal of the American Statistical Association 90, 773-794.

[8] Yang Chengxu, Zheng Yu, Xu Zhoulong. Integrated spatial error modeling of four axis motion platform based on multi-body system theory $[\mathrm{J}]$. Modern manufacturing engineering, 2009 (4): 01-04.

[9] Andreas Pott, Andrés Kecskeméthy, Manfred Hiller. A simplified force-based method for the linearization and sensitivity analysis of complex manipulation systems [J]. 42 (11): 1445-1461. 
[10] Xiao longfan, Du Qun GUI, Wu Lei, et al. Geometric error sensitivity identification of machine tools based on linear response surface method [J]. Modular machine tool and automatic machining technology, 2018 (4): 97-101.

[11] Kang Yueran, Fu Yiyuan, Liu Pengjun, et al. Identification of key error sources of three-axis turntable based on sensitivity analysis [J]. Modular machine tool and automatic machining technology, 2019 (04): 49-52.

[12] LI J, XIE F G, LIU X J. Geometric error modeling and sensitivity analysis of a five-axis machine tool [J]. International Journal of Advanced Manufacturing Technology, 2015, 7 (5): 1.
[13] Fan J, Tang Y, Chen D, et al. A geometric error tracing method based on the Monte Carlo theory of the five-axis gantry machining center $[\mathrm{J}]$. Advances in Mechanical Engineering, 2017, 9 (7): 1687814017707648 -

[14] Xi Chunjiang. Looking at the development trend of international CNC compound internal and external grinder from cimt2019 [J]. World manufacturing technology and equipment market, 2019 (04): 83-85.

[15] Mahbubur Rahman, Jouko Heikkala, Kauko Lappalainen. Modeling, measurement and error compensation of multi-axis machine tools. Part I: theory [J]. International Journal of Machine Tools \& Manufacture, 2000, 40 (10): 1535-1546. 546. 